\title{
Characterization of microRNAs differentially expressed during bovine follicle development
}

\author{
Sadanand D Sontakke, Bushra T Mohammed, Alan S McNeilly ${ }^{1}$ and F Xavier Donadeu \\ The Roslin Institute and Royal (Dick) School of Veterinary Studies, University of Edinburgh, Easter Bush, \\ Midlothian EH25 9RG, UK and ${ }^{1}$ The Queen's Medical Research Institute, MRC Centre for Reproductive Health, \\ 47 Little France Crescent, Edinburgh EH16 4TJ, UK
}

Correspondence should be addressed to F X Donadeu; Email: xavier.donadeu@roslin.ed.ac.uk

\begin{abstract}
Several different miRNAs have been proposed to regulate ovarian follicle function; however, very limited information exists on the spatiotemporal patterns of miRNA expression during follicle development. The objective of this study was to identify, using microarray, miRNA profiles associated with growth and regression of dominant-size follicles in the bovine monovular ovary and to characterize their spatiotemporal distribution during development. The follicles were collected from abattoir ovaries and classified as small (4-8 mm) or large (12-17 mm); the latter were further classified as healthy or atretic based on estradiol and CYP19A1 levels. Six pools of small follicles and individual large healthy $(n=6)$ and large atretic $(n=5)$ follicles were analyzed using Exiqon's miRCURY LNA microRNA Array 6th gen, followed by qPCR validation. A total of 17 and 57 sequences were differentially expressed (greater than or equal to twofold; $P<0.05$ ) between large healthy and each of small and large atretic follicles respectively. Bovine miRNAs confirmed to be upregulated in large healthy follicles relative to small follicles (bta-miR-144, bta-miR-202, bta-miR-451, bta-miR-652, and bta-miR-873) were further characterized. Three of these miRNAs (bta-miR-144, bta-miR-202, and bta-miR-873) were also downregulated in large atretic follicles relative to large healthy follicles. Within the follicle, these miRNAs were predominantly expressed in mural granulosa cells. Further, body-wide screening revealed that bta-miR-202, but not other miRNAs, was expressed exclusively in the gonads. Finally, a total of 1359 predicted targets of the five miRNAs enriched in large healthy follicles were identified, which mapped to signaling pathways involved in follicular cell proliferation, steroidogenesis, prevention of premature luteinization, and oocyte maturation.

Reproduction (2014) $\mathbf{1 4 8} 271-283$
\end{abstract}

\section{Introduction}

In monovular species, including humans, cattle and horses, waves of follicular growth occur periodically in the ovary in response to follicle-stimulating hormone (FSH) throughout a female's reproductive life (Donadeu \& Pedersen 2008). An initial phase of common growth within a wave is followed by the selection of a single follicle, usually the largest (occasionally the two largest), which continues to grow as a dominant follicle while all other follicles (subordinate) cease development and undergo atresia. Subsequent maturation of the dominant follicle may be followed a few days later by ovulation and transformation of the follicle remnants into a corpus luteum. Each of these developmental steps involves sequential and profound changes in tissue structure and function, requiring finely tuned and coordinated changes in the expression of thousands of genes. Transcriptome analyses have identified many such genes during follicle selection, maturation, and the follicle-luteal transition (Gilbert et al. 2011, Rao et al. 2011, Christenson et al. 2013), although relatively little is still known about the molecular mechanisms regulating their expression, particularly at the posttranscriptional level.

miRNAs are key post-transcriptional regulators during cell growth and differentiation (Huntzinger \& Izaurralde 2011). Several studies have already reported effects of specific miRNAs on different aspects of granulosa cell function (Donadeu et al. 2012), including steroidogenesis (Yao et al. 2010, Xu et al. 2011, Yin et al. 2012, Dai et al. 2013), proliferation (Yao et al. 2010, Yan et al. 2012, Dai et al. 2013, Zhang et al. 2013), survival (Carletti et al. 2010, Lin et al. 2012, Yang et al. 2012), terminal differentiation (Kitahara et al. 2013), and cumulus expansion (Yao et al. 2014). With a few exceptions (Carletti et al. 2010, Kitahara et al. 2013), most evidence on follicular roles of miRNAs has been obtained using cultured cells, particularly rodent cells, and in some cases actual changes in the expression of these miRNAs during follicle development have not been demonstrated. Indeed, although genome-wide miRNA analyses of whole ovarian tissues (Landgraf et al. 2007, Ro et al. 2007, Mishima et al. 2008, Hossain et al. 2009, 
Ahn et al. 2010, Juanchich et al. 2013) or follicular, or luteal tissues (Fiedler et al. 2008, Tesfaye et al. 2009, McBride et al. 2012, Miles et al. 2012, da Silveira et al. 2012, Donadeu \& Schauer 2013, Schauer et al. 2013, Sohel et al. 2013) have been reported in several species, detailed spatiotemporal profiles encompassing several follicle developmental stages have not. Identifying such profiles will be an important step toward understanding the functional involvement of miRNAs during specific stages of follicle development.

With this in mind, this study aimed to use microarray analyses to identify miRNA profiles associated with the growth and atresia of dominant-size follicles in bovine and to further characterize the identified miRNAs by establishing their spatiotemporal patterns during development, as well as identify the follicular cell pathways putatively targeted by these miRNAs.

\section{Materials and methods}

\section{Collection and processing of bovine tissues}

All tissues were collected from beef cattle at an abattoir and transported on ice to the laboratory. After rinsing with $70 \%$ ethanol, ovarian pairs containing a visible corpus luteum (Ireland et al. 1980) were selected for dissection. Individual follicles measuring $3-17 \mathrm{~mm}$ in diameter were carefully dissected out and their diameter determined in two different planes using a Vernier caliper. Each follicle was then hemisected and the follicular fluid collected and centrifuged at $800 \mathrm{~g}$ for $10 \mathrm{~min}$. The resulting supernatant was stored at $-80{ }^{\circ} \mathrm{C}$ until further analyses and the cell pellet was combined with the follicular wall free of surrounding stroma and snapped frozen in liquid nitrogen until RNA extraction. In some experiments, after hemi-dissection follicles were gently scraped with blunt-ended forceps to separately collect granulosa cell and theca wall compartments. Theca walls were washed repeatedly to remove any residual granulosa cells. Whenever needed, mural granulosa cells and cumulusoocyte complexes (COCs) were collected separately from follicle pools using a $75 \mu \mathrm{m}$ filter (Fisher Scientific, Loughborough, UK). Follicular components were then separately snapped frozen in liquid nitrogen.

Intrafollicular concentrations of estradiol $\left(\mathrm{E}_{2}\right)$ and progesterone were measured using the competitive double antibody RIA kits (Siemens Healthcare Diagnostics, Inc., Camberley, UK) following manufacturer's instructions. All assays were validated in our laboratory by showing parallelism between serial sample dilutions and the provided assay standard curve (range, $5-500 \mathrm{pg} / \mathrm{ml} \mathrm{E}_{2}$ and $100-4000 \mathrm{pg} / \mathrm{ml}$ progesterone). Sensitivity of the assays was 0.87 and $10 \mathrm{pg} / \mathrm{ml}$, and the intra-assay coefficient of variations were 6 and $4.3 \%$ for $\mathrm{E}_{2}$ and progesterone respectively.

\section{RNA extraction}

Total RNA was extracted from snap-frozen bovine tissues using the miRNeasy Mini Kit (Qiagen, Manchester, UK) following the manufacturer's instructions and previous homogenization with ceramic beads (Lysing Matrix D, MP Biomedicals, Santa Ana, CA, USA) using FastPrep FP120 Cell disruptor (MP Biomedicals). Total RNA was quantified by the NanoDrop-1000 spectrophotometer (NanoDrop Technologies, Willmington, DE, USA) and its quality was determined on the Agilent 2100 Bioanalyzer (Agilent Technologies, Inc., Wokingham, UK).

Follicular fluid was processed following Exiqon's guidelines for plasma RNA purification using the miRNeasy Mini Kit. In brief, $250 \mu \mathrm{l}$ of follicular fluid were centrifuged at $9400 \mathrm{~g}$ for 5 min at $4{ }^{\circ} \mathrm{C}$ to pellet down cellular contents. Two hundred microliter of clear supernatant were taken and mixed with $750 \mu \mathrm{l}$ of QIAzol lysis buffer containing MS2 carrier RNA (Roche Diagnostics Ltd, Burgess Hill, UK), and the mixture was vortexed vigorously for a minute. Following incubation for $5 \mathrm{~min}$ at room temperature, each sample was spiked-in with 5 fmol of synthetic cel-miR-39 (Qiagen) and RNA extracted as per manufacturer's instructions (Qiagen).

\section{Microarray analyses}

Microarray analyses were carried out on pools of follicles measuring 4-8 $\mathrm{mm}$ in diameter (small follicles; six pools, three to five follicles of different diameters per pool) and individual dominant-size follicles $(12-17 \mathrm{~mm})$ that had been previously classified as large healthy $(n=6)$ or large atretic $(n=5)$ based on $\mathrm{E}_{2}$ and CYP19A1 levels (Fig. 1). The microarray procedure was carried out at Exiqon Vedbaek, Denmark. In brief, 600 ng total RNA from each sample were labeled with $\mathrm{Hy} 3$ fluorescent label using the miRCURY LNA microRNA Hi-Power Labeling Kit, Hy3/Hy5 (Exiqon) as per the manufacturer's instructions. A pool of samples was labeled with $\mathrm{Hy} 5$ and used as reference sample. The Hy3-labeled samples and Hy5-labeled reference RNA sample were mixed pairwise and hybridized to the miRCURY LNA microRNA Array 6th gen (Exiqon), which contained 1488 capture probes targeting all microRNAs for human, mouse, or rat registered in miRBase 16.0. The hybridization was carried out according to the miRCURY LNA microRNA Array Instruction manual using a Tecan HS4800 hybridization station (Tecan, Grödig, Austria). After hybridization, the microarray slides were scanned and stored in an ozone-free environment (ozone level below $2.0 \mathrm{ppb}$ ) in order to prevent potential bleaching of the fluorescent dyes. The miRCURY LNA microRNA Array slides were scanned using the Agilent G2565BA Microarray Scanner System and the image analysis was carried out using the ImaGene 9 (miRCURY LNA microRNA Array Analysis Software, Exiqon). The quantified signals were background corrected (Normexp with offset value 10) and normalized using the global Lowess regression algorithm.

The background threshold was calculated for each individual microarray slide as 1.2 times the 25th percentile of the overall signal intensity of the slide. MicroRNAs with intensities above threshold in $<20 \%$ (or 2 ) of the samples were removed from the final dataset used for the expression analysis. The number of present calls obtained (between 460 and 540) was highly comparable among samples.

For each probe and sample, an $M$-value was calculated as the $\log 2$ median of the ratio between $\mathrm{Hy} 3$ and $\mathrm{Hy} 5$ signals for 

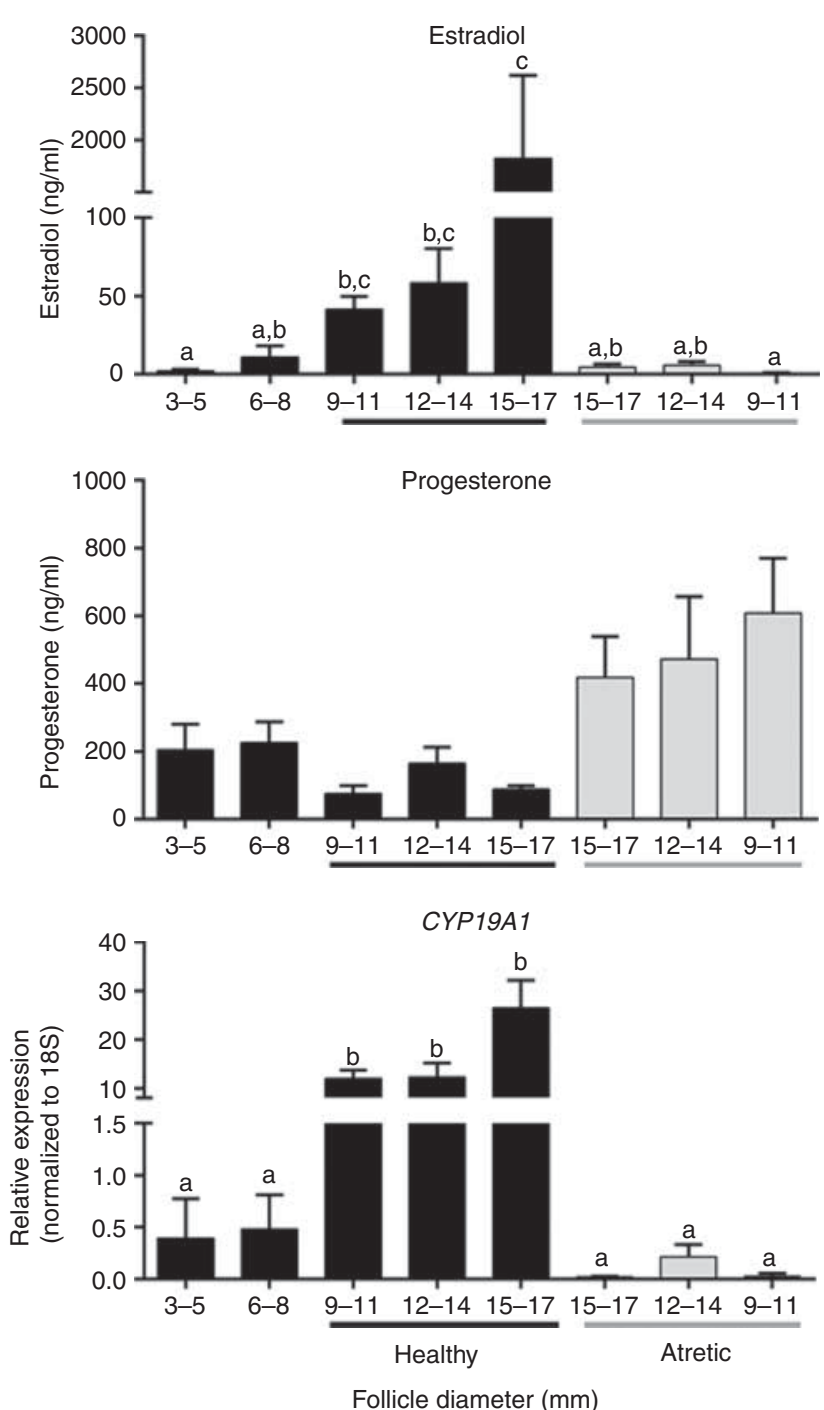

Figure 1 Mean ( \pm s.E.M.) follicular fluid levels of estradiol $\left(E_{2}\right)$ and progesterone, and CYP19A1 transcript levels in whole follicle tissues from cattle ( $n=5-11$ samples/diameter category). There was a significant effect of follicle diameter for $\mathrm{E}_{2}(P<0.0001)$, progesterone $(P=0.02)$, and CYP19A1 $(P<0.0001)$. Means with different superscripts $(\mathrm{a}, \mathrm{b}, \mathrm{c})$ are different $(P<0.05)$.

each of the four spots present on a slide. These values were used to compare the expression of each miRNA probe between large healthy and small follicles and between large healthy and large atretic follicles using Student's $t$-test with Benjamini and Hochberg false discovery rate adjustment $(P<0.05)$.

\section{RT-qPCR}

Total RNA obtained from tissues (500 ng) or cell-free follicular fluid $(6 \mu \mathrm{l})$ was analyzed with miScript II RT and miScript SYBR Green PCR Kits using miScript Primer assays, as per manufacturer's instructions (Qiagen). The MX3005P QPCR System (Stratagene, La Jolla, CA, USA) was used, and a dissociation curve was included at the end of each run to confirm the presence of a single product.
Relative miRNA abundance was obtained using MX3005P Software by extrapolation of $C$ t values from a standard curve prepared from a pool of tissue samples and run simultaneously. Endogenous RnU6-2 or, in the case of follicular fluid, spiked-in cel-miR-39 was used for normalization of miRNA data.

Relative quantification of mRNA levels was carried out on the same cDNA used for miRNA analyses. Bovine-specific primers against CYP19A1 (CGCAAAGCCTTAGAGGATGA and ACCATGGTGATGTACTTTCC), FSHR (TGTGCATTCAATGGAACCCAACTCG and TGACCGGTCCAGAGGCTCCC), CYP17A1 (GGCGACCTTACCATTG and AGATGAGTTGCGTCCC), SPRED1 (ACGAAGCCAAGCCAATCAGA and CСTCCACTTTCCTTGGGCAT), TGFBR2 (GAAGTCCTGCTGGAGCAACT and AGGCAATCTTGGGGTCATGG), ATG7 (GGCCTCTCCAGATTGCAGTT and GCTTCGTCTAGCCGGTACTC), CREB1 (AATCCTAGTGCCCAGCAACC and CGCTGTGCGAATCTGGTATG), and LHCGR (GGACTCTAGCCCGTAGG and ACACATAACCACCATACCAAG) were used with the SensiFAST SYBR Lo-ROX Kit (Bioline Reagents Ltd, London, UK) on the MX3005P QPCR system, as per the manufacturer's instructions. Relative miRNA abundance was calculated as indicated earlier and data were normalized using $18 \mathrm{~S}$ values within each sample.

Dixon's Outlier test was applied to both qPCR and immunoassay data sets, and outlier values $(P<0.01)$ were excluded from subsequent analyses. Non-normal data were log-transformed before analyses. Data were analyzed using the general linear model procedure by one- or two-way ANOVA followed by Tukey's pairwise comparison tests. Data sets involving only two experimental groups were analyzed using Student's t-tests. Spearman's rank correlation test was used to determine the relationship between miRNA levels in whole follicle tissues and follicular fluid. In all cases, statistical significance was considered at $P<0.05$.

\section{In situ hybridization}

The tissues were fixed in 4\% paraformaldehyde (PFA; SigmaAldrich, St. Louis, MO, USA) for $48 \mathrm{~h}$ at $4{ }^{\circ} \mathrm{C}$. After deparaffinization and rehydration, ovarian sections $(5 \mu \mathrm{m})$ were denatured with $5 \mu \mathrm{g} / \mathrm{ml}$ proteinase $\mathrm{K}$ in $75 \mathrm{ml} \mathrm{PBS}$, fixed in $4 \%$ PFA for $10 \mathrm{~min}$ and rinsed with $0.2 \%$ glycine in PBS. The ovarian sections were incubated with freshly prepared imidazole buffer (VWR International Ltd, Lutterworth, UK) and then placed in a humidified chamber. Freshly prepared 1-ethyl-3-(3-dimethylaminopropyl) carbodiimide (SigmaAldrich) was added to each slide for $1 \mathrm{~h}$ at room temperature, followed by $2 \mathrm{~h}$ of prehybridization with $50 \%$ formamide and $5 \times$ SSC buffer at $35^{\circ} \mathrm{C}$. The sections were then incubated overnight with double digoxigenin-labeled LNA-modified oligonucleotide probes against bta-miR-202 (80 nM), RnU62 (3 nM), or scrambled RNA (40 nM) (Exiqon) in a hybridization buffer solution $(50 \%$ formamide, $5 \times$ SSC, $10 \%$ Dextran sulfate salt) in a humidified chamber. After application of the probe, slides were covered with gel bond film and heated at $60^{\circ} \mathrm{C}$ for $5 \mathrm{~min}$. After overnight incubation in a humidifying chamber at $35^{\circ} \mathrm{C}$, the slides were washed with $2 \% \mathrm{BSA}$ and $0.2 \times \mathrm{SSC}$ post hybridization buffer for $10 \mathrm{~min}$ three times at $4{ }^{\circ} \mathrm{C}$ to avoid unspecific binding, followed by 


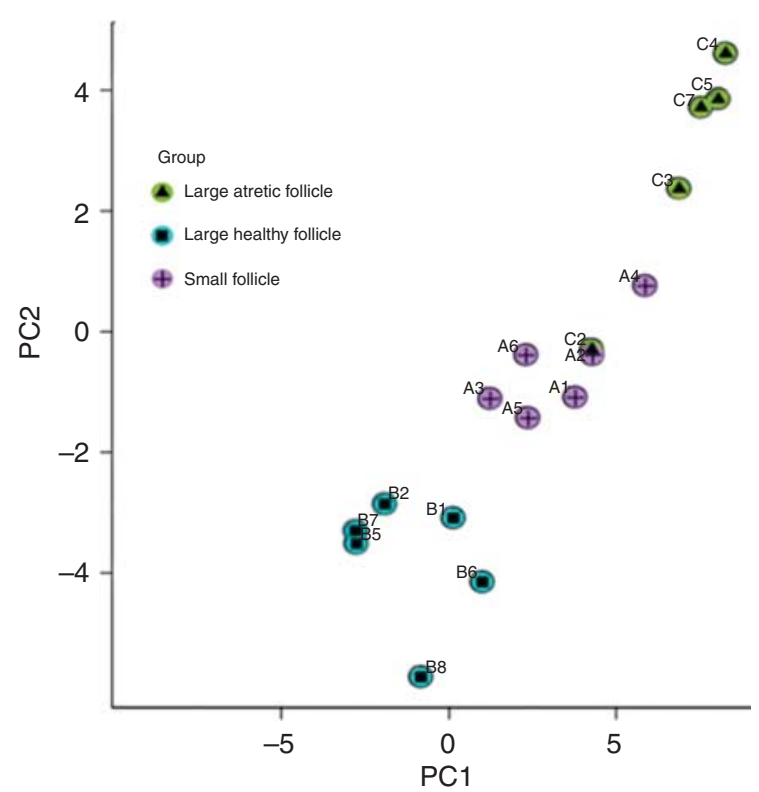

Figure 2 Principal component analyses plot performed on all follicular samples used for microarray, and on the top 50 microRNAs with the highest S.D. The normalized log ratio values were used for the analysis. The features were shifted to be zero centered, (i.e. the mean value across samples was shifted to zero) and scaled to have unit variance (i.e. the variance across samples was scaled to one before the analysis).

rinsing with $1 \times$ TBS. The slides were then incubated with $2 \%$ sheep serum and $1 \%$ BSA for $1 \mathrm{~h}$ at room temperature followed by incubation with anti-digoxigenin antibody $(1: 200$; Roche) for $2 \mathrm{~h}$ at room temperature, and color development with NBT/BCIP (Roche) at $4{ }^{\circ} \mathrm{C}$ for up to $16 \mathrm{~h}$. The samples were observed under light microscope at $20 \times$ (Leica 800). Three independent analyses were carried out using different ovarian sections.

\section{Granulosa cell culture}

Granulosa cells collected from follicles measuring $4-8 \mathrm{~mm}$ were passed through a filter mesh $(70 \mu \mathrm{m}$; Fisher Scientific) to separate out COCs. The cells were then treated with red blood cell lysis buffer $(8.3 \mathrm{gm} / \mathrm{l} \mathrm{NH} 4 \mathrm{Cl}$ in $0.01 \mathrm{M}$ Tris- $\mathrm{HCl}$ buffer of $\mathrm{pH}$ 7.5), washed with fresh McCoy's 5a medium, and cultured in 96-well plates (Fisher Scientific) with modified McCoy's 5a medium containing 0.02 M HEPES, $3 \mathrm{mM}$ L-glutamine, $0.1 \%$ BSA, and $1 \%$ Pen-Strep, and supplemented with $10 \mathrm{ng} / \mathrm{ml}$ bovine insulin, $2.5 \mathrm{mg} / \mathrm{l}$ transferrin, $4 \mu \mathrm{g} / \mathrm{l}$ sodium selenite, and $100 \mathrm{nM}$ androstenedione (Gutierrez et al. 1997) in a humidified atmosphere at $37{ }^{\circ} \mathrm{C}$ with $5 \% \mathrm{CO}_{2}$. Twenty-four hours after plating, the cells were left untreated or were treated with Forskolin $(10 \mathrm{mM})$, bovine insulin $(1 \mathrm{mg} / \mathrm{ml})$, and FCS $(1 \%, \mathrm{v} / \mathrm{v})$ for up to 6 days, with media replacement every 2 days. On different days during culture, the cells were harvested and processed for quantification of miRNA levels as described earlier. Three different experiments with cultured granulosa cells were carried out.

\section{Results}

A total of 94 antral follicles were collected and analyzed. As expected, the levels of $E_{2}$ and CYP19A1 transcript were higher in follicles $\geq 9 \mathrm{~mm}$ (dominant-size follicles classified as healthy) than in follicles $3-8 \mathrm{~mm}$ in diameter (Fig. 1). $\mathrm{E}_{2}$ and CYP19A1 levels remained low in a subset of $\geq 9 \mathrm{~mm}$ follicles, classified as atretic, which also had relatively high mean levels of progesterone.

\section{Microarray analyses}

A total of 545 probes yielded hybridization intensities above the background threshold across the follicular samples analyzed. These corresponded to 523 unique miRNAs including 191 sequences registered as bovine in miRBase release 18 . The results of comparative analyses of follicular groups (large healthy vs small and large healthy vs large atretic) are shown in Supplementary Table 1, see section on supplementary data given at the end of this article (raw microarray data have been deposited in the NCBI's GEO repository, GSE54692). Principal Component Analysis revealed clustering of samples largely according to follicle class (Fig. 2). A total of 17 and 57 miRNA sequences were differentially expressed (greater than or equal to twofold; adjusted $P$ value $<0.05)$ between large healthy and small follicles and between large healthy and large atretic follicles respectively (Tables 1 and 2). Out of these, eight and 20 miRNAs, respectively, corresponded to bovine sequences registered in miRBase. A total of seven miRNAs were upregulated in large healthy follicles relative to small follicles; these miRNAs were also expressed at higher levels in large healthy than in large atretic follicles. Moreover, out of a total of ten miRNAs

Table 1 miRNAs differentially expressed (greater than or equal to twofold) between small and large healthy follicles in bovine.

\begin{tabular}{lcc}
\hline miRNA & $\begin{array}{c}\text { Fold } \\
\text { change }\end{array}$ & $\begin{array}{c}\text { Adj. } \\
\text { value }^{\mathrm{a}}\end{array}$ \\
\hline $\begin{array}{l}\text { Downregulated in large healthy follicle } \\
\text { hsa-miR-3178 }\end{array}$ & \\
hsa-miR-1246/bta-miR-1246 & 3.60 & 0.002 \\
hsa-miR-1290 & 2.97 & 0.027 \\
hsa-miR-1275 & 2.82 & 0.035 \\
hsa-miR-625-3p & 2.29 & 0.020 \\
hsa-miR-3621 & 2.21 & 0.006 \\
hsa-miR-483-3p/bta-miR-483 & 2.17 & 0.002 \\
hsa-miR-1469 & 2.17 & 0.002 \\
hsa-miR-498 & 2.15 & 0.014 \\
hsa-miR-4279 & 2.14 & 0.003 \\
Upregulated in large healthy follicle & 2.05 & 0.012 \\
bta-miR-202 & & \\
hsa-miR-876-5p/bta-miR-876 & 4.18 & 0.002 \\
hsa-miR-876-3p & 3.79 & 0.002 \\
hsa-miR-873-5p/bta-miR-873 & 3.09 & 0.002 \\
hsa-miR-451a/bta-miR-451 & 2.70 & 0.003 \\
hsa-miR-144-3p/bta-miR-144 & 2.65 & 0.014 \\
hsa-miR-652-3p/bta-miR-652 & 2.35 & 0.009 \\
\hline
\end{tabular}

${ }^{\mathrm{a}} \mathrm{FDR}$; Benjamini and Hochberg adjustment. 
downregulated in large healthy follicles relative to small follicles, eight were also downregulated relative to large atretic follicles. Of all sequences upregulated between

Table 2 miRNAs differentially expressed (greater than or equal to twofold) between large atretic follicles and large healthy follicles in bovine.

\begin{tabular}{|c|c|c|}
\hline miRNA & $\begin{array}{c}\text { Fold } \\
\text { change }\end{array}$ & $\begin{array}{c}\text { Adj. } \\
\text { P value }^{\mathrm{a}}\end{array}$ \\
\hline \multicolumn{3}{|c|}{ Downregulated in large healthy follicle } \\
\hline hsa-miR-659-3p & 6.05 & 0.000 \\
\hline hsa-miR-4285 & 5.69 & 0.001 \\
\hline hsa-miR-1469 & 5.11 & 0.001 \\
\hline hsa-miR-625-3p & 4.94 & 0.000 \\
\hline hsa-miR-642b-3p & 4.62 & 0.004 \\
\hline hsa-miR-765 & 4.11 & 0.001 \\
\hline hsa-miR-675-5p & 4.08 & 0.000 \\
\hline hsa-miR-422a & 3.89 & 0.000 \\
\hline hsa-miR-498 & 3.69 & 0.000 \\
\hline hsa-miR-665 & 3.66 & 0.003 \\
\hline hsa-miR-483-3p/bta-miR-483 & 3.64 & 0.001 \\
\hline hsa-miR-1909-3p & 3.61 & 0.001 \\
\hline hsa-miR-3178 & 3.36 & 0.001 \\
\hline hsa-miR-149-3p & 3.12 & 0.001 \\
\hline hsa-miR-3685 & 3.11 & 0.006 \\
\hline bta-miR-21* & 3.09 & 0.002 \\
\hline hsa-miR-2116-5p & 3.09 & 0.001 \\
\hline hsa-miR-4279 & 2.96 & 0.001 \\
\hline hsa-miR-551b-5p & 2.85 & 0.000 \\
\hline hsa-miR-1236 & 2.83 & 0.000 \\
\hline hsa-miR-4258 & 2.78 & 0.000 \\
\hline hsa-miR-373-5p & 2.74 & 0.000 \\
\hline hsa-miR-1915-3p & 2.71 & 0.000 \\
\hline hsa-miR-4292 & 2.58 & 0.001 \\
\hline hsa-miR-150-5p/bta-miR-150 & 2.54 & 0.001 \\
\hline hsa-miR-371a-5p & 2.50 & 0.001 \\
\hline bta-miR-21 & 2.39 & 0.001 \\
\hline hsa-miR-204-3p & 2.36 & 0.000 \\
\hline bta-miR-409a & 2.36 & 0.000 \\
\hline hsa-miR-744-5p/bta-miR-744 & 2.36 & 0.002 \\
\hline hsa-miR-4306 & 2.34 & 0.002 \\
\hline hsa-miR-1275 & 2.28 & 0.003 \\
\hline hsa-miR-361-3p & 2.27 & 0.001 \\
\hline hsa-miR-3621 & 2.21 & 0.001 \\
\hline hsa-miR-638 & 2.18 & 0.000 \\
\hline hsa-miR-4268 & 2.17 & 0.001 \\
\hline hsa-miR-3676-3p & 2.15 & 0.000 \\
\hline hsa-miR-2115-3p & 2.09 & 0.002 \\
\hline hsa-miR-142-5p/bta-miR-142 & 2.03 & 0.001 \\
\hline \multicolumn{3}{|c|}{ Upregulated in large healthy follicle } \\
\hline bta-miR-202 & 9.98 & 0.000 \\
\hline hsa-miR-876-5p/bta-miR-876 & 5.99 & 0.000 \\
\hline hsa-miR-31-3p & 4.90 & 0.001 \\
\hline hsa-miR-31-5p/bta-miR-31 & 3.94 & 0.001 \\
\hline hsa-miR-876-3p & 3.92 & 0.000 \\
\hline hsa-miR-873-5p/bta-miR-873 & 3.53 & 0.000 \\
\hline hsa-miR-652-3p/bta-miR-652 & 3.50 & 0.000 \\
\hline hsa-miR-450b-5p & 3.17 & 0.012 \\
\hline hsa-miR-210/bta-miR-210 & 2.98 & 0.002 \\
\hline hsa-miR-451a/bta-miR-451 & 2.49 & 0.031 \\
\hline bta-miR-34b & 2.22 & 0.028 \\
\hline hsa-miR-190a/bta-miR-190a & 2.10 & 0.004 \\
\hline hsa-miR-15b-5p/bta-miR-15b & 2.10 & 0.006 \\
\hline hsa-miR-424-5p & 2.07 & 0.033 \\
\hline hsa-miR-4275 & 2.06 & 0.001 \\
\hline hsa-miR-33a-5p/bta-miR-33a & 2.04 & 0.005 \\
\hline hsa-miR-144-3p/bta-miR-144 & 2.02 & 0.032 \\
\hline hsa-miR-487b/bta-miR-487b & 2.01 & 0.003 \\
\hline
\end{tabular}

${ }^{\mathrm{a}} \mathrm{FDR}$; Benjamini and Hochberg adjustment. large healthy and small follicles all but one corresponded to bona-fide bovine miRNAs (bta-miR-144, bta-miR-202, bta-miR-451, bta-miR-652, bta-miR-873, and bta-miRNA-876) and thus this set of miRNAs (Table 3) was chosen for further characterization.

\section{Validation of microarray data by $q P C R$}

Confirmatory qPCR analyses were carried out across a wide range of developmental stages to more precisely identify the follicle diameter categories involved in the differences in miRNA expression observed by microarray. These analyses revealed an increase in the expression of bta-miR-144, bta-miR-202, bta-miR-451, bta-miR-652, and bta-miR-873 in large healthy relative to small follicles (Fig. 3A), in agreement with the results of microarray analyses. A significant increase in the levels of these miRNAs occurred at the 12-14 mm stage or later, except for miR-873 for which expression levels increased above fourfold between 6-8 and 9-11 mm follicles. A decrease in expression levels between large healthy and large atretic follicles involving one or more diameter categories for each of these follicle types was confirmed for three of the five miRNAs analyzed (miR-144, miR-202, and miR-873). The expression of bta-miR-876 was presumably too low in bovine follicles and could not be detected by qPCR.

Changes in the expression of selected miRNAs (btamiR-483, bta-miR-150, bta-miR-21, bta-miR-409a, btamiR-378, and bta-miR-142) that were present at lower levels in large healthy follicles than in large atretic follicles (Table 2 and Supplementary Table 1) were also validated by qPCR using samples from the follicles measuring 9-17 mm in diameter at different stages of growth or atresia (Fig. 3B). For five of the six miRNAs analyzed, the results of qPCR were in agreement with those of microarray, in that their levels were significantly higher in all or some of the large atretic follicle categories relative to large Healthy follicles. An exception was bta-miR-483, for which levels were not significantly different between large healthy and large atretic follicles.

\section{Localization of miRNAs within follicular compartments}

We next sought to determine the localization of miRNAs within follicular compartments. Granulosa (including COCs) and theca fractions were separately collected from individual healthy or atretic follicles measuring 9-17 mm in diameter (Fig. 4). Relative abundance of FSHR and CYP17A1 transcripts in each cell fraction was used to ensure the lack of cross-contamination between fractions collected from the same follicle (Fig. 4A). As shown in Fig. 4B, three of the five miRNAs upregulated in large healthy follicles relative to small follicles, such as miR-144, miR-202, and miR-873, were expressed at 
Table 3 Bovine miRNAs sequences upregulated (greater than or equal to twofold) between large healthy follicle and small follicles.

\begin{tabular}{llccc}
\hline miRNA & Mature sequence & Chromosome & Genomic location start-end (strand) & Locus property \\
\hline bta-miR-202 & UUCCUAUGCAUAUACUUCUUU & 26 & $25988240-25988338(+)$ & Intergenic \\
bta-miR-876 & UGGAUUUCUUUGUGAAUCACCA & 8 & $15183224-15183304(+)$ & Intergenic \\
bta-miR-873 & GCAGGAACUUGUGAGUCUCCU & 8 & $15159560-15159636(+)$ & Intergenic \\
bta-miR-451 & AAACCGUUACCAUUACUGAGUUU & 19 & $20796860-20796930(-)$ & Intergenic \\
bta-miR-144 & UACAGUAUAGAUGAUGUACUAG & 19 & $20797022-20797105(-)$ & Intergenic \\
bta-miR-652 & AAUGGCGCCACUAGGGUUGUG & $X$ & $62939283-62939380(+)$ & $T M E M 164$, intron 1 \\
\hline
\end{tabular}

much higher levels in granulosa cells than in theca cells. In contrasts, miR-652 was expressed at similar levels in the two cell types whereas miR-451 was present at slightly higher levels in theca cells. To determine whether, as presumed, the enrichment of miRNAs in the granulosa compartment reflected the expression by mural granulosa cells rather than by COCs, we analyzed these two compartments in small and large follicles. The results (Fig. 4C) showed that an increase in the levels of all five miRNAs in large follicles could indeed be attributed to a large extent to an increase in miRNA expression in mural granulosa cells rather than in the COC.

To confirm the results of qPCR, we then carried out in situ hybridization of bta-miR-202. We chose this miRNA as it has been reported to be expressed specifically in the ovary in other species and moreover its expression within bovine follicles appeared to be clearly compartmentalized (Fig. 4B). Consistent with qPCR data, the miR-202 probe hybridized most intensely to granulosa cells, with some signal also detected in the theca compartment but not in the ovarian stroma (Fig. 5A). miR-202 had a more diffuse cellular distribution than the nucleolar positive control, RnU6-2; however, in contrast to RnU6-2, the miR-202 signal was strongest around the nucleus (Fig. 5A), indicating a high miRNA abundance in the scant granulosa cell cytoplasm, as expected.

To determine whether, as shown in other studies (da Silveira et al. 2012), changes in follicular cell expression of miRNAs would be reflected by follicular fluid levels, the levels of bta-miR-144, bta-miR-202, bta-miR-451, bta-miR-652, and bta-miR-873 were compared between matched samples of fluid and whole tissue from large healthy and large atretic follicles. Owing to limiting follicular fluid volumes, small follicles could not be included in these analyses. Moreover, follicular fluid levels of bta-miR-144 and bta-miR-451 were not detected. Of the other three miRNAs, only the levels of bta-miR-202 were significantly correlated $(P<0.01)$, albeit moderately $(r=0.46)$, between whole follicle tissues and follicular fluid (data not shown).

\section{Body-wide expression of miRNAs}

QPCR screening of an array of body tissues (Fig. 6) showed that bta-miR-144, bta-miR-451, bta-miR-652, and bta-miR-873 were present in tissues across the bovine body, with levels of all these miRNAs except
bta-miR-873 being relatively low in the ovary. In contrast, bta-miR-202 was not expressed in tissues other than testis and ovary, consistent with results in other species (Landgraf et al. 2007, Ro et al. 2007, Armisen et al. 2009, Juanchich et al. 2013).

\section{Functional analyses}

To identify cellular processes putatively targeted by miRNAs in large healthy follicles, lists of predicted bovine gene targets were obtained from TargetScan (release 6.2) and analyzed using Ingenuity Pathway Analysis (http://www.ingenuity.com/). A total of 1359 transcripts were identified, with bta-miR-144 having the highest number of predicted targets (836) followed by bta-miR-873 (302), bta-miR-202 (191), bta-miR-451 (22), and bta-miR-652 (8). Results of ontology analyses of the entire set of target genes are given in Supplementary Table 2, see section on supplementary data given at the end of this article.

We then sought to determine the expression profiles of selected miRNA target genes across follicle development stages. We chose four genes (SPRED1, TGFRB2, ATG7, and CREB1) that were predicted (Supplementary Table 3, see section on supplementary data given at the end of this article) to be simultaneously targeted by two or more of the miRNAs, bta-miR-144, bta-miR-202, and bta-miR873, whose expression most dynamically changed across follicular stages (Fig. 3A). The expression of all of SPRED1, TGFRB2, ATG7, and CREB1 was significantly lower in large healthy than in small or large atretic follicles, whereas the levels of the three miRNAs were highest in large healthy follicles (Fig. 7). These profiles were consistent with SPRED1, TGFRB2, ATG7, and CREB1 being the targets of bta-miR-144, bta-miR202, and bta-miR-873 in large healthy follicles. In contrast, levels of LHCGR, a predicted nontarget, were highest in large healthy follicles and lowest in large atretic follicles, as expected.

To more precisely investigate the actual functions of miRNAs in follicular cells, we first tested whether miRNA expression patterns during follicle development (Fig. 3) could be modeled in vitro. Granulosa cells from small follicles were cultured under conditions that prevent early luteinization (Gutierrez et al. 1997) and 1 day later cells were treated with Forskolin to induce differentiation or were left untreated, as in previous in vitro miRNA validation studies (McBride et al. 2012). 

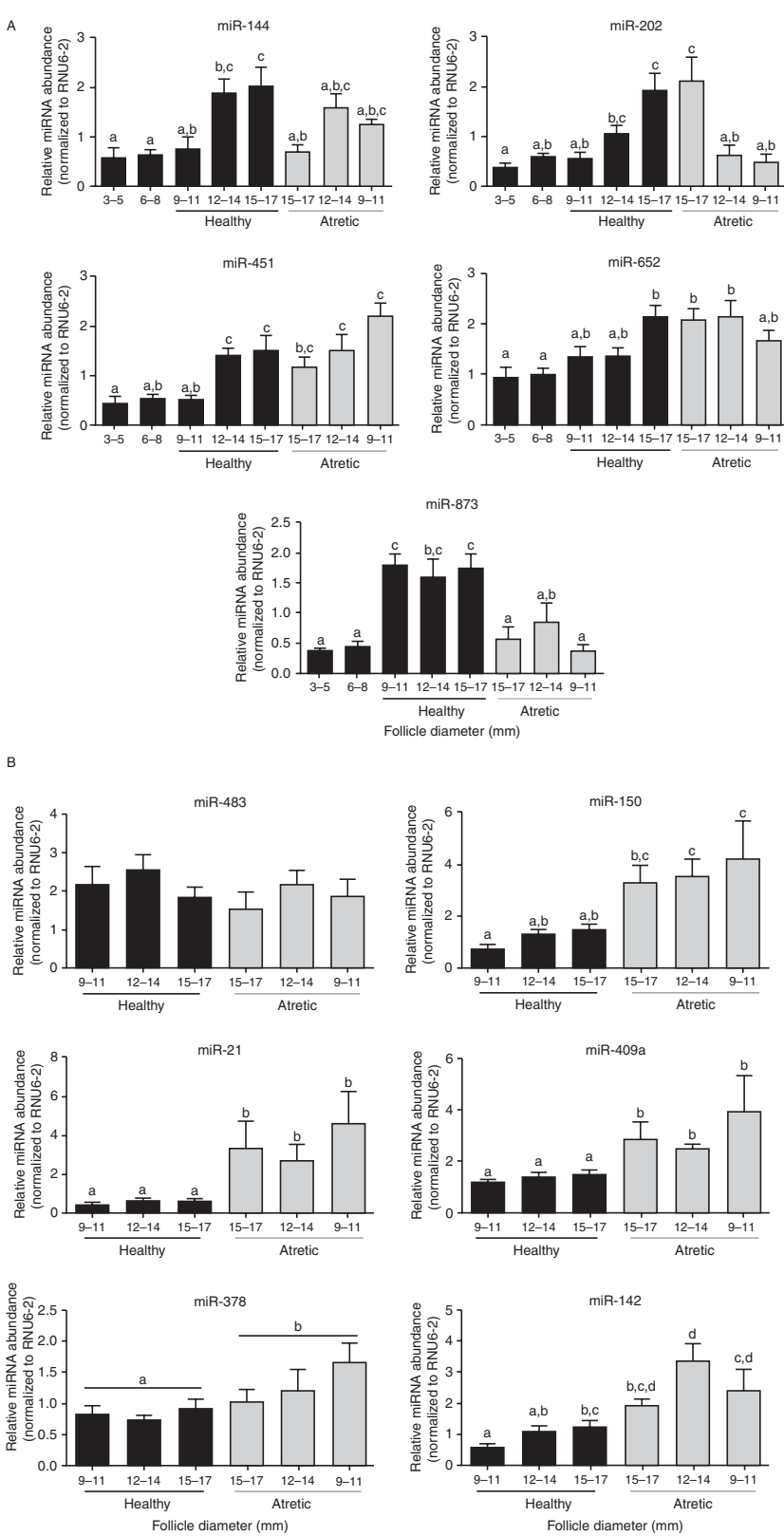

Figure 3 Relative abundance (mean \pm s.E.M.) of miRNAs in bovine follicles ( $n=4-11$ samples/diameter category). Bovine miRNAs whose expression was upregulated in large healthy follicle relative to small follicles in the microarray analyses are shown in (A) and selected miRNAs whose expression was downregulated in large healthy follicles relative to large atretic follicles are shown in (B). There was a main effect of follicle diameter $(P<0.01)$ for all miRNAs except bta-miR-483 $(P>0.1)$. Means with different superscripts $(a, b, c)$ are different $(P<0.05)$.

Among those miRNAs upregulated in large healthy follicles, the expression of bta-miR-144, bta-652, and bta-miR-873 quickly decreased upon culture and their levels were consistently nondetected after 1-2 days. In contrast, the levels of bta-miR-202 and bta-miR-451 were readily detectable by qPCR for up to 6 days of culture; however, treatment with Forskolin did not result in an increase in the levels of either miRNA in granulosa cells (Fig. 8). We also determined in granulosa cell cultures the expression of some of the miRNAs that were downregulated in large healthy follicles relative to large atretic follicles, namely, bta-miR-409a, bta-miR-150, and bta-miR-21. We found that although the levels
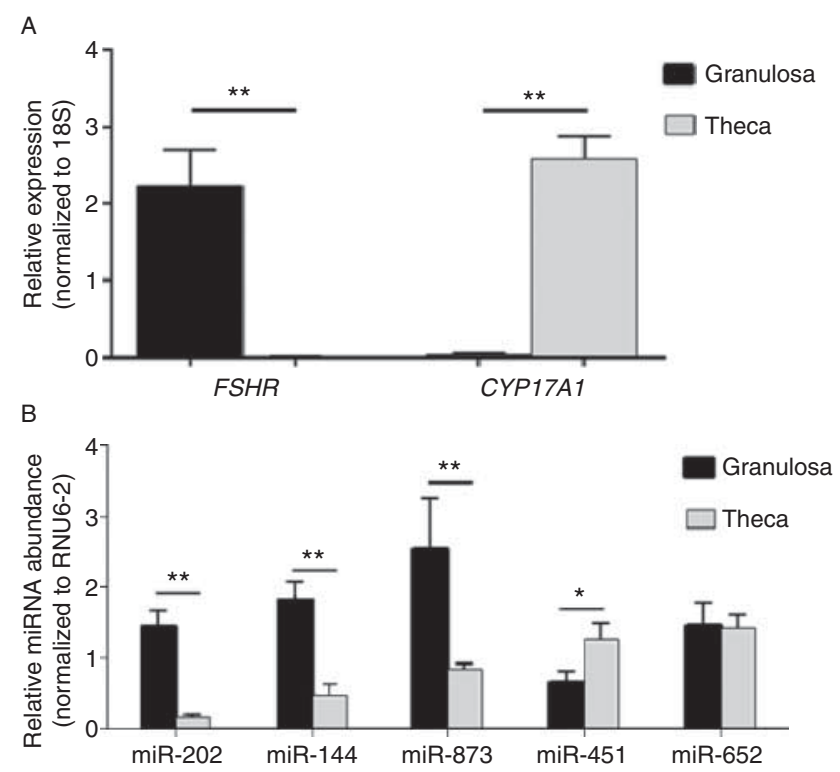

C
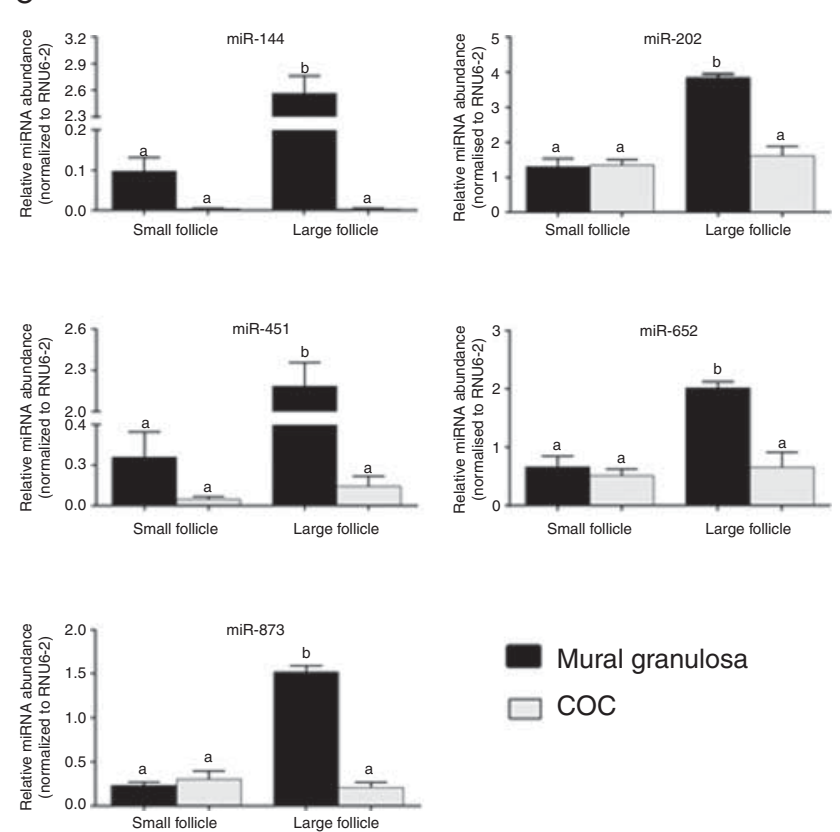

Mural granulosa

COC

Figure 4 Relative abundance (mean \pm S.E.M.) of (A) FSHR and CYP17A1 and (B) miRNAs in granulosa and theca cell fractions of bovine healthy follicles (9-17 mm; $n=10$ follicles). Mean differences are indicated by $* P<0.05$ or $* * P<0.01$. (C) Relative abundance of the same miRNAs in mural granulosa and cumulus-oocyte complex fractions from small (4$8 \mathrm{~mm}$ ) and large $(9-17 \mathrm{~mm})$ follicles $(n=4-6$ pools/follicle group, three to five samples per pool). There was an interaction of cell fraction $X$ follicle category for all miRNAs $(P<0.001)$. Means with different superscripts $(\mathrm{a}, \mathrm{b})$ are different $(P<0.05)$. 


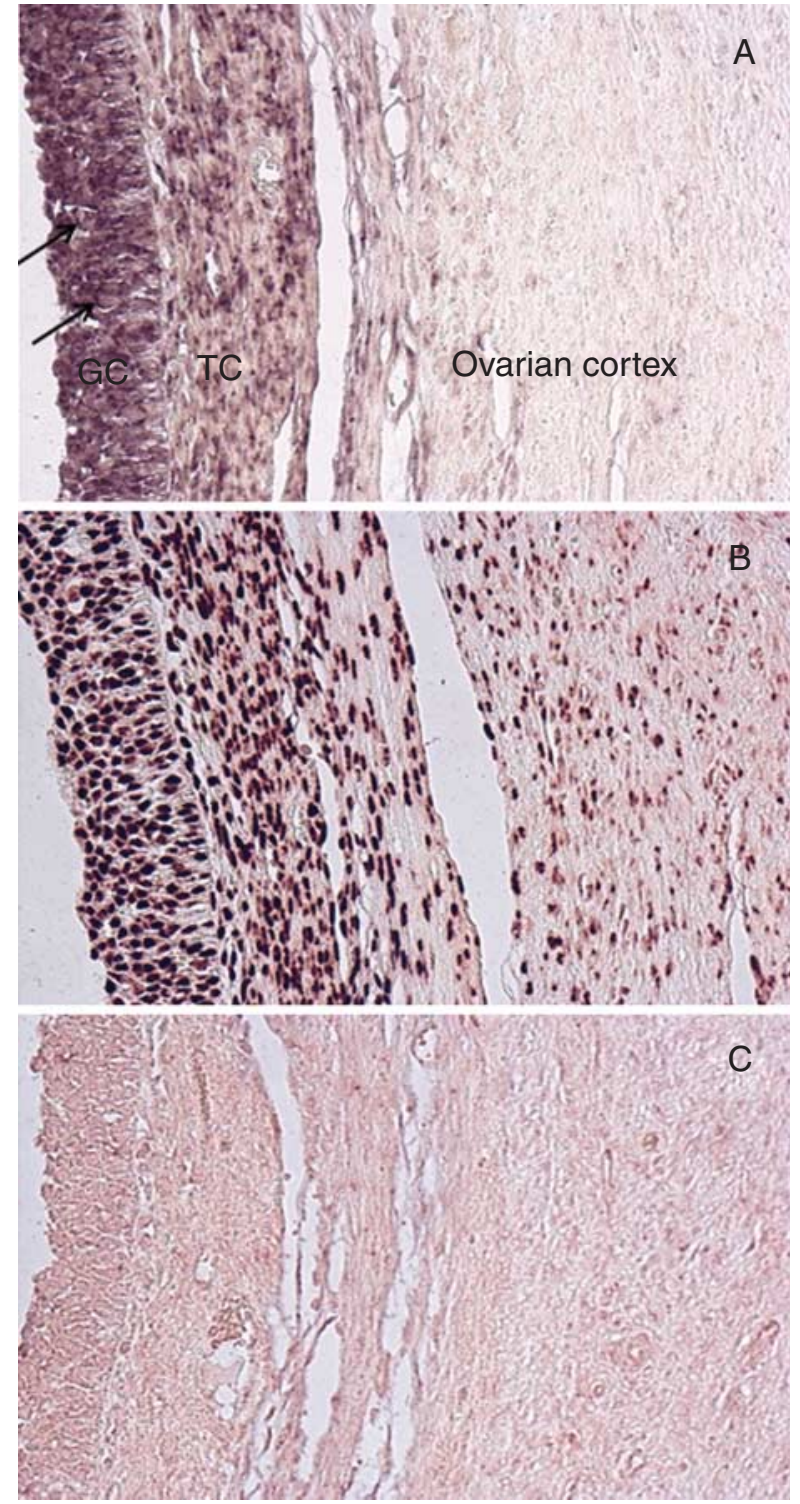

Figure 5 Representative images showing results of in situ hybridization of bta-miR-202 in bovine follicular wall. Formalin-fixed paraffin embedded ovary sections were hybridized with DIG-labeled LNA probes for bta-miR-202 (A), the small nucleolar RNA, RnU6-2 (B), or a scrambled oligonucleotide sequence $(C)$. Perinuclear areas with strong miR-202 hybridization in granulosa cells are indicated by arrows. GC and TC indicate the granulosa and theca compartments of the follicular wall.

of bta-miR-409a were quickly downregulated upon culture, bta-miR-150 and bta-miR-21 continued to be expressed and either did not change or, in the case of bta-miR-150, decreased overtime in response to Forskolin (Fig. 8). These results, while preventing further in vitro functional analyses of miRNAs enriched in large healthy follicles, also indicated that, overall, miRNA expression may be regulated differently in vivo and in vitro, as already reported for miR-21 and other miRNAs (Carletti et al. 2010, McBride et al. 2012).

\section{Discussion}

An involvement in the regulation of follicle function has been proposed for several miRNAs (reviewed in Donadeu et al. (2012)), although most evidence to date derives from results using granulosa cell cultures. Moreover, physiological changes in the expression of miRNAs supporting putative roles during follicle development have often not been demonstrated (Hu et al. 2012, Yang et al. 2012, Dai et al. 2013), and detailed spatiotemporal miRNA profiles during development are not available. In a previous study (McBride et al. 2012), we used sequencing to characterize the developmental changes in miRNA expression in ovine follicles, although our focus was on terminal differentiation (follicle-luteal transition) rather than on follicle growth and atresia. In this regard, the present findings are important as they provide novel information on the dynamic changes in miRNA levels across a wide range of follicle developmental stages in a monovular species, the cow, identifying a subset of miRNAs that are putatively involved in growth and regression of dominant follicles.

Our first objective was to use expression profiling to identify miRNA populations uniquely associated with steroidogenically active dominant-size follicles. We reasoned that by comparing miRNA profiles between large healthy follicles and each of small and large atretic follicles we would identify subsets of miRNAs putatively involved in follicle growth and atresia respectively. Using a heterologous microarray approach we were able to profile a total of 191 known bovine miRNAs in follicular tissues. In a previous study using sequencing, we identified a total of 212 unique miRNAs across different stages of follicle and luteal development in sheep (McBride et al. 2012), whereas Fiedler et al. (2008) earlier detected 206 different miRNA transcripts by microarray analyses of mouse granulosa cells.

Relative to large healthy follicles, a significantly larger number of sequences were differentially expressed in large atretic follicles than that in small follicles, although only a fraction of these corresponded to registered bovine miRNAs (Tables 1 and 2). Many of the bovine miRNAs differentially expressed in large atretic follicles are reportedly involved in regulating the proliferation and survival of different cell types (miR-483, miR-150, miR-21, miR-409a, miR-31, miR-34b, miR-33a; Yamakuchi et al. 2008, Bertero et al. 2011, Liu et al. 2011, Brabletz 2012, Cirera-Salinas et al. 2012, Weng et al. 2012) as well as immune cell activation (miR-150, miR-21, miR-31; Xiao et al. 2007, Xue et al. 2013, Das et al. 2014), which is consistent with an active role of these miRNAs in follicular fate decisions. The involvement of miR-21 in the promotion of cell survival is well established, including in mouse granulosa cells during ovulation (Carletti et al. 2010). In this study, miR-21 levels were on average sixfold higher in bovine large atretic follicle than that in large healthy follicles (Fig. 3B). Interestingly, earlier expression profiling of equine 

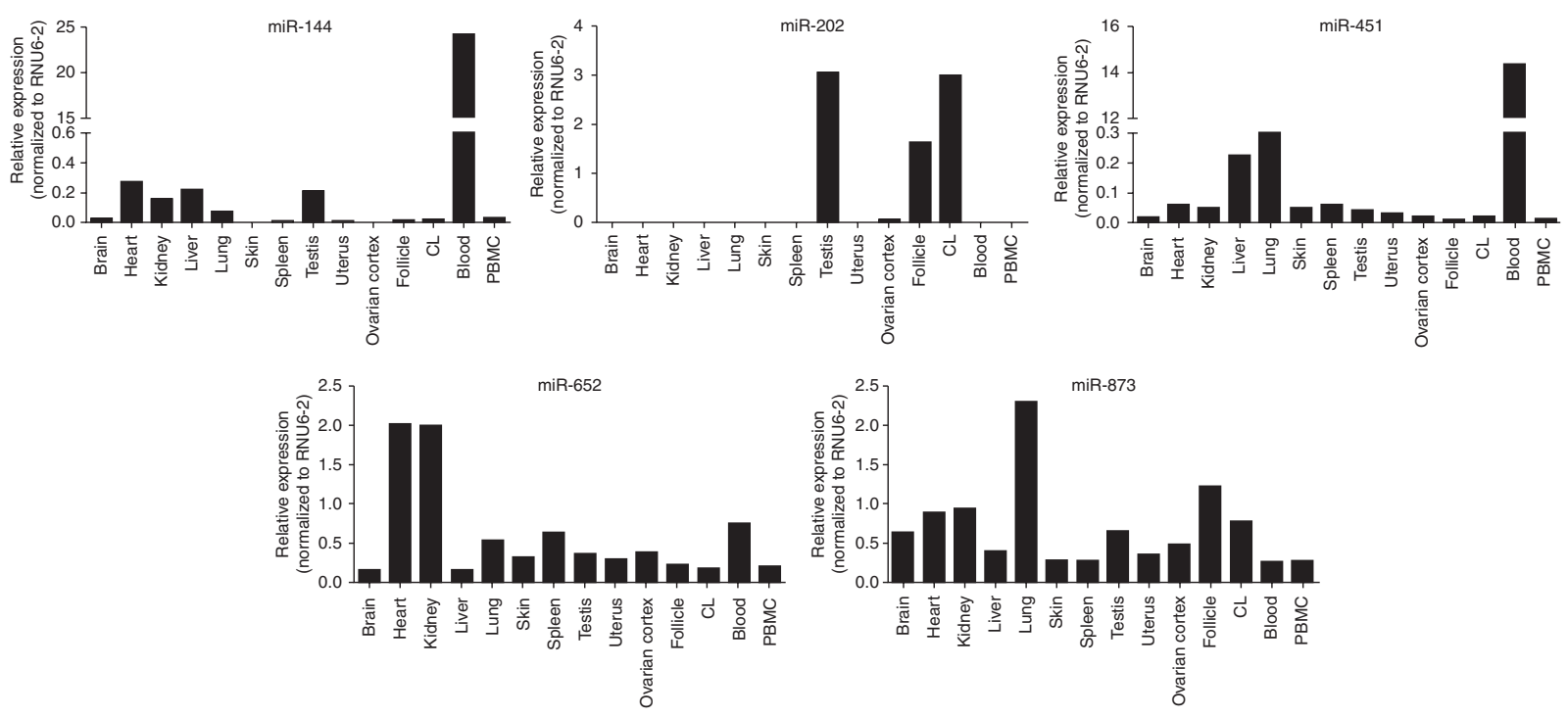

Figure 6 Relative abundance of miRNAs in pools of different bovine tissues (minimum of three animals per pool). CL, corpus luteum; PBMC, peripheral blood mononuclear cells.

follicular fluid found increased mean miR-21 levels in subordinate follicles relative to dominant follicles (Schauer et al. 2013), and in seasonally anovulatory follicles relative to ovulatory follicles (Donadeu \& Schauer 2013). Taken together, these data suggest a role for miR-21 in follicular atresia; in light of previous information showing an involvement of this miRNA in the mediation of immune and fibrotic responses in other body systems (Kumarswamy et al. 2011), the role of miR-21 in follicular death warrants further investigation.

Many of the differences in miRNA expression between large healthy follicles and small follicles were also found
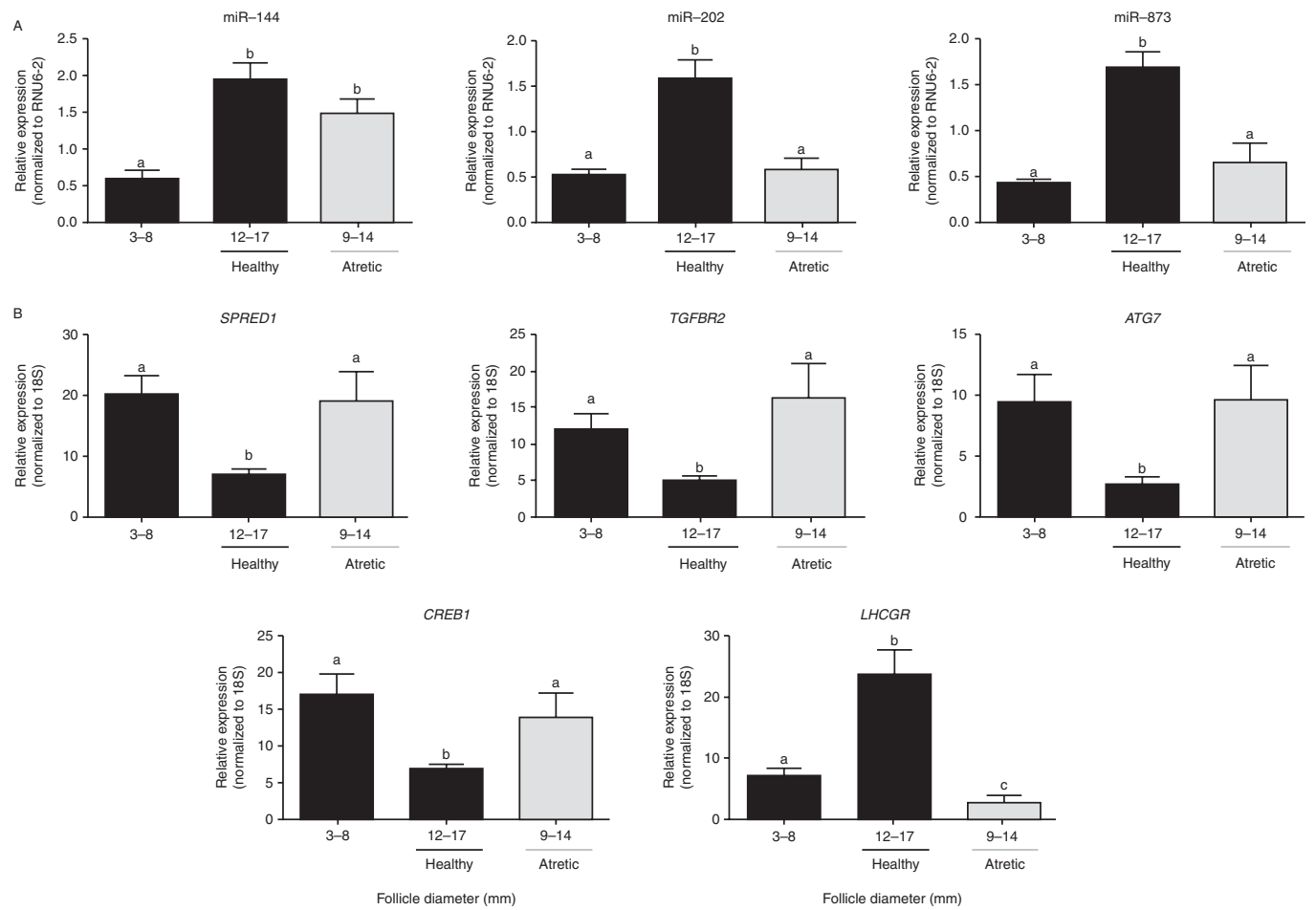

Figure 7 Relative abundance (mean \pm S.E.M.) across different categories of bovine follicles ( $n=9-19$ samples/diameter category) of (A) miR-144, miR-202, and miR-873 and (B) selected transcripts (SPRED1, TGFBR2, ATG7, and CREB1) predicted to be targeted by those miRNAs, as well as a predicted nontarget $(L H C G R)$. There was an effect of follicle category $(P<0.01)$ for all endpoints. Means with different superscripts $(a, b, c)$ are different $(P<0.05)$. 

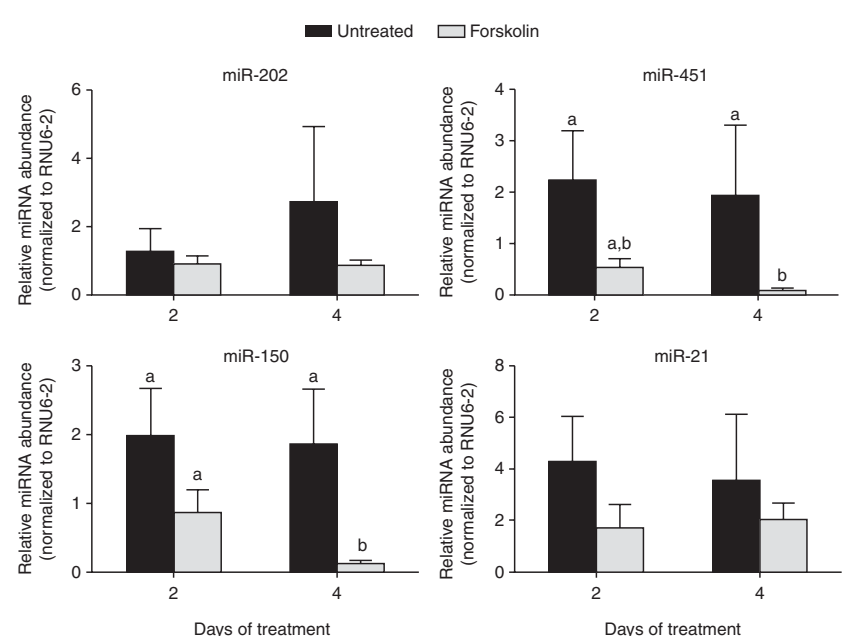

Figure 8 Changes in the levels (mean \pm s.E.M.) of miR-202, miR-451, miR-150, and miR-21 in bovine granulosa cells cultured in the presence of Forskolin $(10 \mu \mathrm{M})$, insulin $(1 \mu \mathrm{g} / \mathrm{ml})$, and FCS $(1 \%)$ or left untreated for 4 days. Fold changes in miRNA levels relative to levels before the onset of treatment (day 0 ) are shown. There was an interaction of treatment $\times$ day for miR-451 $(P=0.04)$ and miR-150 $(P=0.005)$. Means with different superscripts $(a, b)$ are different $(P<0.05)$.

between large healthy follicles and large atretic follicles. Principal component analyses showed three clearly distinct follicle populations; however, because the health status of small follicles could not be determined, samples within this category might have presumably contained atretic as well as healthy follicles, which could account for some of the similarities in miRNA patterns between the small follicles and large atretic follicle groups. Most of the sequences upregulated in large healthy follicles relative to small follicles corresponded to bona-fide bovine miRNAs (bta-miR-144, bta-miR-202, bta-miR-451, bta-miR-652, and bta-miR873). QPCR analyses confirmed that at least three of these miRNAs were also upregulated significantly in some or all of the large healthy follicle categories relative to large atretic follicles, thus identifying this set of miRNAs as very strong candidates with a role in the development of bovine dominant follicles. mir-144, mir-202, mir-451, and mir-652 were reported earlier to be expressed in the ovary (Hossain et al. 2009, McBride et al. 2012, Kitahara et al. 2013, Schauer et al. 2013), although their expression patterns within follicular tissues were not studied in detail. In this study, bta-miR-202 was restricted to the bovine gonad; moreover, its expression within the follicle was highest in granulosa cells while levels in ovarian stroma were negligible. These findings are consistent with data showing gonad-restricted expression of mir-202 in humans, rodents, frogs, and fish (Landgraf et al. 2007, Ro et al. 2007, Armisen et al. 2009, Juanchich et al. 2013) and the expression of mir-202 predominantly in Sertoli cells within the mouse testis (Wainwright et al. 2013). Studies on chicken and mice showed sexually dimorphic expression of mir-202 in primordial gonads under regulation of estrogen or Sox9, suggesting a role in early testis development. The present result of a greater than fourfold increase in bta-miR-202 levels between 9-11 and 15-17 mm healthy follicles builds on those earlier findings by indicating a role for this miRNA during folliculogenesis in the adult bovine ovary. In addition to mir-202, biological roles for the clustered mir-144 and mir-451 have also been proposed, but only in relation to non-ovarian cells; those include regulatory roles in erythropoiesis (Pase et al. 2009), differentiation of vascular smooth muscle cells (Turczyńska et al. 2013) and, potentially relevant during follicular differentiation, cholesterol trafficking in macrophages (Ramirez et al. 2013). Interestingly, both miR-144 and miR-451, as well as miR-652, were upregulated in rat ovaries following an ovulatory hCG bolus, although no attempt was made to identify the specific cell type(s) involved (Kitahara et al. 2013).

To gain insights into their spatiotemporal involvement during follicle development, the expression of bta-miR-144, bta-miR-202, bta-miR-451, bta-miR-652, and bta-miR873 was determined over a wide range of developmental stages and cell compartments in bovine follicles, providing information comparatively more detailed than in any previous study. An increase in the levels of all of these miRNAs in large follicles was accounted for increases in mural granulosa cells rather than in COC; this was not surprising as the latter contains a very small fraction of the total cells in the follicle, and therefore microarray analyses of whole follicle tissues might have missed changes in miRNA levels in the COC. Indeed, miRNAs differentially expressed in bovine COCs, cumulus cells or oocytes, in addition to changes in miRNA expression during oocyte maturation, have been reported (Hossain et al. 2009, Tesfaye et al. 2009, Miles et al. 2012), and they did not include any of the miRNAs identified as enriched in large healthy follicles in this study. Nonetheless, our results suggest a potential involvement of bta-miR-144, bta-miR-202, bta-miR451, bta-miR-652, and bta-miR-873 in the regulation of mural granulosa cell function during maturation of the dominant follicle, as their expression in all cases increased in healthy follicles once they reached a diameter of 12-14 mm. In contrast to the other miRNAs, a sharp increase in the levels of bta-miR-873 occurred as early as the 9-11 mm stage in healthy follicles and was associated with a significant reduction in bta-miR-873 levels in all large atretic follicle categories; these temporal relationships suggest a potential role of this miRNA in selection of the dominant follicle, a possibility that warrants further investigation, particularly because of the lack of functional data on miR-873 in any cell type or species.

To identify cell functions and pathways potentially targeted by bta-miR-144, bta-miR-202, bta-miR-451, btamiR-652 and bta-miR-873 in dominant follicles, we 
carried out ontology analyses for the complete set of predicted mRNA targets obtained by TargetScan (Supplementary Table 2). Many of the signaling pathways overrepresented by these targets corresponded to growth factors putatively involved in follicular cell proliferation and steroidogenesis and prevention of premature luteinization including HGF, IGF1, TGF $\beta$, estrogen, neurotropin/TRK, PEDF, and NGF (Parrott et al. 1993, Salas et al. 2006, Glister et al. 2010, Mani et al. 2010, Garcia-Rudaz et al. 2011, Chuderland et al. 2013) and others involved in oocyte maturation including neuregulin, GDNF, and EGF (Conti et al. 2006, Noma et al. 2011, Linher-Melville \& Li 2013), the fine-tune regulation of which is of key importance during the final stages of follicle development. Also present were several core cell cycle regulatory pathways (cell cycle regulation by BTG family proteins, estrogen-mediated S phase entry, and G1/S checkpoint regulation), a finding which is consistent with the notion that the development of the dominant follicle involves a substantial decrease in granulosa cell proliferation (Lussier et al. 1987, Gaytan et al. 1996).

Bioinformatics analyses were complemented by demonstrating that the changes in the levels of selected transcripts during follicle development were consistent with these being the targets of miRNAs in large healthy follicles, specifically, a decrease in the expression of TGFBR2 and CREB1, two transcripts involved in TGF $\beta$ and cAMP signaling and which are involved in follicular differentiation (Somers et al. 1995, Wehrenberg et al. 1998), and SPRED1 and ATG7, two transcripts involved in RAS/MAPK signaling and autophagy, respectively, and which expression during follicular development has not been previously characterized. Unfortunately, changes in miRNA expression during bovine follicle development could not be replicated in cultures of granulosa cells, which prevented further in vitro validation of genes and signaling pathways putatively targeted by miRNAs. Nonetheless, our results provide useful information by showing that bta-miR-144, bta-miR-202, bta-miR-45, bta-miR-652, and miR-873 may in combination play a key role in the final maturation of the bovine dominant follicle in preparation for the subsequent follicle-luteal transition.

In summary, this study identified for the first time genome-wide miRNA profiles associated with growth and atresia of dominant-size follicles in bovine. Five miRNAs were upregulated in large healthy follicle relative to small follicle categories (bta-miR-144, bta-miR-202, bta-miR-451, bta-miR-652, and bta-miR873) and three of these (bta-miR-144, bta-miR-202, and bta-miR-873) were also expressed at higher levels in large healthy follicle than in large atretic follicle categories. These three miRNAs were predominantly expressed in the mural granulosa cell compartment of the follicle, and one of them, bta-miR-202, was not expressed in body tissues other than the gonad. Finally, gene ontology analyses of predicted miRNA targets revealed that, within the growing dominant follicle, these miRNAs putatively target multiple signaling pathways involved in follicular cell proliferation, steroidogenesis, and prevention of premature luteinization, in addition to oocyte maturation. In conclusion, the present data identify a specific set of miRNAs that may play a key role during the maturation of dominant follicles in the bovine ovary.

\section{Supplementary data}

This is linked to the online version of the paper at http://dx.doi. org/10.1530/REP-14-0140.

\section{Declaration of interest}

The authors declare that there is no conflict of interest that could be perceived as prejudicing the impartiality of the research reported.

\section{Funding}

S D Sontakke and B T Mohammed were supported by a Commonwealth PhD scholarship and Kurdistan Regional scholarship funds respectively. The Roslin Institute receives strategic grant funding from the BBSRC.

\section{Acknowledgements}

The authors thank Stephanie Schauer and Shriram Bhosle for technical assistance with qPCR and IPA analysis.

\section{References}

Ahn HW, Morin RD, Zhao H, Harris RA, Coarfa C, Chen ZJ, Milosavljevic A, Marra MA \& Rajkovic A 2010 MicroRNA transcriptome in the newborn mouse ovaries determined by massive parallel sequencing. Molecular Human Reproduction 16 463-471. (doi:10. 1093/molehr/gaq017)

Armisen J, Gilchrist MJ, Wilczynska A, Standart N \& Miska EA 2009 Abundant and dynamically expressed miRNAs, piRNAs, and other small RNAs in the vertebrate Xenopus tropicalis. Genome Research 19 1766-1775. (doi:10.1101/gr.093054.109)

Bertero T, Gastaldi C, Bourget-Ponzio I, Imbert V, Loubat A, Selva E, Busca R, Mari B, Hofman P, Barbry P et al. 2011 miR-483-3p controls proliferation in wounded epithelial cells. FASEB Journal 25 3092-3105. (doi:10.1096/fj.10-168401)

Brabletz T 2012 miR-34 and SNAIL: another double-negative feedback loop controlling cellular plasticity/EMT governed by p53. Cell Cycle $\mathbf{1 1}$ 215-216. (doi:10.4161/cc.11.2.18900)

Carletti MZ, Fiedler SD \& Christenson LK 2010 MicroRNA 21 blocks apoptosis in mouse periovulatory granulosa cells. Biology of Reproduction 83 286-295. (doi:10.1095/biolreprod.109.081448)

Christenson LK, Gunewardena S, Hong X, Spitschak M, Baufeld A \& Vanselow J 2013 Research resource: preovulatory LH surge effects on follicular theca and granulosa transcriptomes. Molecular Endocrinology 27 1153-1171. (doi:10.1210/me.2013-1093)

Chuderland D, Ben-Ami I, Kaplan-Kraicer R, Grossman H, Komsky A, Satchi-Fainaro R, Eldar-Boock A, Ron-El R \& Shalgi R 2013 Hormonal regulation of pigment epithelium-derived factor (PEDF) in granulosa cells. Molecular Human Reproduction 19 72-81. (doi:10.1093/molehr/ gas046) 
Cirera-Salinas D, Pauta M, Allen RM, Salerno AG, Ramírez CM, ChamorroJorganes A, Wanschel AC, Lasuncion MA, Morales-Ruiz M, Suarez $Y$ et al. 2012 Mir-33 regulates cell proliferation and cell cycle progression. Cell Cycle 11 922-933. (doi:10.4161/cc.11.5.19421)

Conti M, Hsieh M, Park JY \& Su YQ 2006 Role of the epidermal growth factor network in ovarian follicles. Molecular Endocrinology 20 715-723. (doi:10.1210/me.2005-0185)

Dai A, Sun H, Fang T, Zhang Q, Wu S, Jiang Y, Ding L, Yan G \& Hu Y 2013 MicroRNA-133b stimulates ovarian estradiol synthesis by targeting Foxl2. FEBS Letters 587 2474-2482. (doi:10.1016/j.febslet.2013.06.023)

Das A, Ganesh K, Khanna S, Sen CK \& Roy S 2014 Engulfment of apoptotic cells by macrophages: a role of microRNA-21 in the resolution of wound inflammation. Journal of Immunology 192 1120-1129. (doi:10.4049/ jimmunol.1300613)

Donadeu FX \& Pedersen HG 2008 Follicle development in mares. Reproduction in Domestic Animals 43 (Suppl 2) 224-231. (doi:10.1111/j. 1439-0531.2008.01166.x)

Donadeu FX \& Schauer SN 2013 Differential miRNA expression between equine ovulatory and anovulatory follicles. Domestic Animal Endocrinology 45 122-125. (doi:10.1016/j.domaniend.2013.06.006)

Donadeu FX, Schauer SN \& Sontakke SD 2012 Involvement of miRNAs in ovarian follicular and luteal development. Journal of Endocrinology 215 323-334. (doi:10.1530/JOE-12-0252)

Fiedler SD, Carletti MZ, Hong X \& Christenson LK 2008 Hormonal regulation of microRNA expression in periovulatory mouse mural granulosa cells. Biology of Reproduction 79 1030-1037. (doi:10.1095/ biolreprod.108.069690)

Garcia-Rudaz C, Dorfman M, Nagalla S, Svechnikov K, Söder O, Ojeda SR \& Dissen GA 2011 Excessive ovarian production of nerve growth factor elicits granulosa cell apoptosis by setting in motion a tumor necrosis factor $\alpha /$ stathmin-mediated death signaling pathway. Reproduction 142 319-331. (doi:10.1530/REP-11-0134)

Gaytan F, Morales C, Bellido C, Aguilar E \& Sanchez-Criado JE 1996 Proliferative activity in the different ovarian compartments in cycling rats estimated by the 5-bromodeoxyuridine technique. Biology of Reproduction 54 1356-1365. (doi:10.1095/biolreprod54.6.1356)

Gilbert I, Robert C, Dieleman S, Blondin P \& Sirard MA 2011 Transcriptional effect of the LH surge in bovine granulosa cells during the peri-ovulation period. Reproduction 141 193-205. (doi:10.1530/REP-10-0381)

Glister C, Satchell L \& Knight PG 2010 Changes in expression of bone morphogenetic proteins (BMPs), their receptors and inhibin co-receptor betaglycan during bovine antral follicle development: inhibin can antagonize the suppressive effect of BMPs on thecal androgen production. Reproduction 140 699-712. (doi:10.1530/REP-10-0216)

Gutierrez C, Campbell B \& Webb R 1997 Development of a long-term bovine granulosa cell culture system: induction and maintenance of estradiol production, response to follicle-stimulating hormone, and morphological characteristics. Biology of Reproduction 56 608-616. (doi:10.1095/biolreprod56.3.608)

Hossain MM, Ghanem N, Hoelker M, Rings F, Phatsara C, Tholen E, Schellander K \& Tesfaye D 2009 Identification and characterization of miRNAs expressed in the bovine ovary. BMC Genomics 10 443. (doi:10. 1186/1471-2164-10-443)

Hu Z, Shen WJ, Kraemer FB \& Azhar S 2012 MicroRNAs 125a and 455 repress lipoprotein-supported steroidogenesis by targeting scavenger receptor class B type I in steroidogenic cells. Molecular and Cellular Biology 32 5035-5045. (doi:10.1128/MCB.01002-12)

Huntzinger E \& Izaurralde E 2011 Gene silencing by microRNAs: contributions of translational repression and mRNA decay. Nature Reviews. Genetics 12 99-110. (doi:10.1038/nrg2936)

Ireland JJ, Murphee RL \& Coulson PB 1980 Accuracy of predicting stages of bovine estrous cycle by gross appearance of the corpus luteum. Journal of Dairy Science 63 155-160. (doi:10.3168/jds.S00220302(80)82901-8)

Juanchich A, Le Cam A, Montfort J, Guiguen Y \& Bobe J 2013 Identification of differentially expressed miRNAs and their potential targets during fish ovarian development. Biology of Reproduction 88 128. (doi:10.1095/ biolreprod.112.105361)

Kitahara Y, Nakamura K, Kogure K \& Minegishi T 2013 Role of microRNA136-3p on the expression of luteinizing hormone-human chorionic gonadotropin receptor mRNA in rat ovaries. Biology of Reproduction $\mathbf{8 9}$ 114. (doi:10.1095/biolreprod.113.109207)
Kumarswamy R, Volkmann I \& Thum T 2011 Regulation and function of miRNA-21 in health and disease. RNA Biology 8 706-713. (doi:10.4161/rna.8.5.16154)

Landgraf $P$, Rusu M, Sheridan R, Sewer A, lovino N, Aravin A, Pfeffer S, Rice A, Kamphorst AO, Landthaler M et al. 2007 A mammalian microRNA expression atlas based on small RNA library sequencing. Cell 129 1401-1414. (doi:10.1016/j.cell.2007.04.040)

Lin F, Li R, Pan ZX, Zhou B, Yu de B, Wang XG, Ma XS, Han J, Shen M \& Liu HL 2012 miR-26b promotes granulosa cell apoptosis by targeting ATM during follicular atresia in porcine ovary. PLOS ONE 7 e38640. (doi:10.1371/journal.pone.0038640)

Linher-Melville K \& Li J 2013 The roles of glial cell line-derived neurotrophic factor, brain-derived neurotrophic factor and nerve growth factor during the final stage of folliculogenesis: a focus on oocyte maturation. Reproduction 145 R43-R54. (doi:10.1530/REP-12-0219)

Liu LZ, Li C, Chen Q, Jing Y, Carpenter R, Jiang Y, Kung HF, Lai L \& Jiang BH 2011 MiR-21 induced angiogenesis through AKT and ERK activation and HIF-1 $\alpha$ expression. PLOS ONE 6 e19139. (doi:10.1371/journal. pone.0019139)

Lussier JG, Matton P \& Dufour JJ 1987 Growth rates of follicles in the ovary of the cow. Journal of Reproduction and Fertility 81 301-307. (doi:10.1530/jrf.0.0810301)

Mani AM, Fenwick MA, Cheng Z, Sharma MK, Singh D \& Wathes DC 2010 IGF1 induces up-regulation of steroidogenic and apoptotic regulatory genes via activation of phosphatidylinositol-dependent kinase/AKT in bovine granulosa cells. Reproduction 139 139-151. (doi:10.1530/REP09-0050)

McBride D, Carre W, Sontakke SD, Hogg CO, Law A, Donadeu FX \& Clinton M 2012 Identification of miRNAs associated with the follicularluteal transition in the ruminant ovary. Reproduction 144 221-233. (doi:10.1530/REP-12-0025)

Miles JR, McDaneld TG, Wiedmann RT, Cushman RA, Echternkamp SE, Vallet JL \& Smith TP 2012 MicroRNA expression profile in bovine cumulus-oocyte complexes: possible role of let-7 and miR-106a in the development of bovine oocytes. Animal Reproduction Science 130 16-26. (doi:10.1016/j.anireprosci.2011.12.021)

Mishima T, Takizawa T, Luo SS, Ishibashi O, Kawahigashi Y, Mizuguchi $Y$, Ishikawa T, Mori M, Kanda T, Goto T et al. 2008 MicroRNA (miRNA) cloning analysis reveals sex differences in miRNA expression profiles between adult mouse testis and ovary. Reproduction 136 811-822. (doi:10.1530/REP-08-0349)

Noma N, Kawashima I, Fan HY, Fujita Y, Kawai T, Tomoda Y, Mihara T, Richards JS \& Shimada M 2011 LH-induced neuregulin 1 (NRG1) type III transcripts control granulosa cell differentiation and oocyte maturation. Molecular Endocrinology 25 104-116. (doi:10.1210/me.2010-0225)

Parrott JA, Whaley PD \& Skinner MK 1993 Extrahepatic expression of fibrinogen by granulosa cells: potential role in ovulation. Endocrinology 133 1645-1649.

Pase L, Layton JE, Kloosterman WP, Carradice D, Waterhouse PM \& Lieschke G) 2009 miR-451 regulates zebrafish erythroid maturation in vivo via its target gata2. Blood 113 1794-1804. (doi:10.1182/blood2008-05-155812)

Ramirez CM, Rotllan N, Vlassov AV, Davalos A, Li M, Goedeke L, Aranda JF, Cirera-Salinas D, Araldi E, Salerno A et al. 2013 Control of cholesterol metabolism and plasma high-density lipoprotein levels by microRNA-144. Circulation Research 112 1592-1601. (doi:10.1161/ CIRCRESAHA.112.300626)

Rao JU, Shah KB, Puttaiah J \& Rudraiah M 2011 Gene expression profiling of preovulatory follicle in the buffalo cow: effects of increased IGF-I concentration on periovulatory events. PLoS ONE 6 e20754. (doi:10. 1371/journal.pone.0020754)

Ro S, Song R, Park C, Zheng H, Sanders K \& Yan W 2007 Cloning and expression profiling of small RNAs expressed in the mouse ovary. RNA 13 2366-2380. (doi:10.1261/rna.754207)

Salas C, Julio-Pieper M, Valladares M, Pommer R, Vega M, Mastronardi C, Kerr B, Ojeda SR, Lara HE \& Romero C 2006 Nerve growth factordependent activation of trkA receptors in the human ovary results in synthesis of follicle-stimulating hormone receptors and estrogen secretion. Journal of Clinical Endocrinology and Metabolism 91 2396-2403. (doi:10.1210/jc.2005-1925) 
Schauer SN, Sontakke SD, Watson ED, Esteves CL \& Donadeu FX 2013 Involvement of miRNAs in equine follicle development. Reproduction 146 273-282. (doi:10.1530/REP-13-0107)

da Silveira JC, Veeramachaneni DN, Winger QA, Carnevale EM \& Bouma GJ 2012 Cell-secreted vesicles in equine ovarian follicular fluid contain miRNAs and proteins: a possible new form of cell communication within the ovarian follicle. Biology of Reproduction 8671. (doi:10.1095/biolreprod.111.093252)

Sohel MM, Hoelker M, Noferesti SS, Salilew-Wondim D, Tholen E, Looft C, Rings F, Uddin MJ, Spencer TE, Schellander K et al. 2013 Exosomal and non-exosomal transport of extra-cellular microRNAs in follicular fluid: implications for bovine oocyte developmental competence. PLOS ONE 8 e78505. (doi:10.1371/journal.pone.0078505)

Somers JP, Benyo DF, Little-Ihrig L \& Zeleznik AJ 1995 Luteinization in primates is accompanied by loss of a 43-kilodalton adenosine $3^{\prime}, 5^{\prime}$-monophosphate response element-binding protein isoform. Endocrinology 136 4762-4768.

Tesfaye D, Worku D, Rings F, Phatsara C, Tholen E, Schellander K \& Hoelker M 2009 Identification and expression profiling of microRNAs during bovine oocyte maturation using heterologous approach. Molecular Reproduction and Development 76 665-677. (doi:10.1002/mrd.21005)

Turczyńska KM, Bhattachariya A, Säll J, Göransson O, Swärd K, Hellstrand P \& Albinsson S 2013 Stretch-sensitive down-regulation of the miR-144/451 cluster in vascular smooth muscle and its role in AMPactivated protein kinase signaling. PLOS ONE 8 e65135. (doi:10.1371/ journal.pone.0065135)

Wainwright EN, Jorgensen JS, Kim Y, Truong V, Bagheri-Fam S, Davidson T, Svingen T, Fernandez-Valverde SL, McClelland KS, Taft RJ et al. 2013 SOX9 regulates microRNA miR-202-5p/3p expression during mouse testis differentiation. Biology of Reproduction 89 34. (doi:10.1095/ biolreprod.113.110155)

Wehrenberg U, Giebel J \& Rune GM 1998 Possible involvement of transforming growth factor- $\beta 1$ and transforming growth factor- $\beta$ receptor type II during luteinization in the marmoset ovary. Tissue \& Cell $\mathbf{3 0}$ 360-367. (doi:10.1016/S0040-8166(98)80049-9)

Weng C, Dong H, Chen G, Zhai Y, Bai R, Hu H, Lu L \& Xu Z 2012 miR-409$3 p$ inhibits HT1080 cell proliferation, vascularization and metastasis by targeting angiogenin. Cancer Letters 323 171-179. (doi:10.1016/ j.canlet.2012.04.010)

Xiao C, Calado DP, Galler G, Thai T-H, Patterson HC, Wang J, Rajewsky N, Bender TP \& Rajewsky K 2007 MiR-150 controls B cell differentiation by targeting the transcription factor c-Myb. Cell 131 146-159. (doi:10. 1016/j.cell.2007.07.021)
Xu S, Linher-Melville K, Yang BB, Wu D \& Li J 2011 Micro-RNA378 (miR378) regulates ovarian estradiol production by targeting aromatase. Endocrinology 152 3941-3951. (doi:10.1210/en.2011-1147)

Xue F, Li H, Zhang J, Lu J, Xia Y \& Xia Q 2013 miR-31 regulates interleukin 2 and kinase suppressor of ras 2 during $\mathrm{T}$ cell activation. Genes and Immunity 14 127-131. (doi:10.1038/gene.2012.58)

Yamakuchi M, Ferlito M \& Lowenstein CJ 2008 miR-34a repression of SIRT1 regulates apoptosis. PNAS 105 13421-13426. (doi:10.1073/pnas. 0801613105)

Yan G, Zhang L, Fang T, Zhang Q, Wu S, Jiang Y, Sun H \& Hu Y 2012 MicroRNA-145 suppresses mouse granulosa cell proliferation by targeting activin receptor IB. FEBS Letters 586 3263-3270. (doi:10. 1016/j.febslet.2012.06.048)

Yang X, Zhou Y, Peng S, Wu L, Lin H-Y, Wang S \& Wang H 2012 Differentially expressed plasma microRNAs in premature ovarian failure patients and the potential regulatory function of mir-23a in granulosa cell apoptosis. Reproduction 144 235-244. (doi:10.1530/REP-11-0371)

Yao G, Yin M, Lian J, Tian H, Liu L, Li X \& Sun F 2010 MicroRNA-224 is involved in transforming growth factor- $\beta$-mediated mouse granulosa cell proliferation and granulosa cell function by targeting Smad4. Molecular Endocrinology 24 540-551. (doi:10.1210/me.2009-0432)

Yao G, Liang M, Liang N, Yin M, Lu M, Lian J, Wang Y \& Sun F 2014 MicroRNA-224 is involved in the regulation of mouse cumulus expansion by targeting Ptx3. Molecular and Cellular Endocrinology 382 244-253. (doi:10.1016/j.mce.2013.10.014)

Yin M, Lu M, Yao G, Tian H, Lian J, Liu L, Liang M, Wang Y \& Sun F 2012 Transactivation of microRNA-383 by steroidogenic factor- 1 promotes estradiol release from mouse ovarian granulosa cells by targeting RBMS1. Molecular Endocrinology 26 1129-1143. (doi:10.1210/me. 2011-1341)

Zhang Q, Sun H, Jiang Y, Ding L, Wu S, Fang T, Yan G \& Hu Y 2013 MicroRNA-181a suppresses mouse granulosa cell proliferation by targeting activin receptor IIA. PLOS ONE 8 e59667. (doi:10.1371/ journal.pone.0059667)

Received 11 March 2014

First decision 31 March 2014

Revised manuscript received 20 May 2014

Accepted 11 June 2014 